\title{
NANOTECHNOLOGY IN FOOD SYSTEMS: A REVIEW
}

\author{
É. KIss* \\ Laboratory of Interfaces and Nanostructures, Institute of Chemistry, Eötvös Loránd University, H-1117 Budapest, \\ Pázmány P. s. 1/a. Hungary
}

(Received: 30 March 2020; accepted: 30 June 2020)

\begin{abstract}
Recent developments of nanotechnology find its way into various fields of food production in our days. Nanotechnology could offer benefits in development of food products with enhanced functionality for health promotion, or modified texture convenient for elderly, and in quality and safety issues in the food supply chain. Nanoencapsulated bioactive components such as vitamins, antibacterial agents contribute to production of enriched food stuffs with the required appearance, flavour, taste, and texture. Nanomaterials can protect the sensitive compounds from environmental attack, release them in a programmed way, and provide favourable improvement in the bioavailability of nutraceuticals. The innovative approach in food packaging, including the detection, indication, and control of food products, serves the quality and safety improvements.

Keywords: enriched food, enhanced quality, intelligent food packaging, nanoencapsulation, vitamins, antimicrobials
\end{abstract}

In the last decade of the 20th century, the conversion of scientific knowledge collected by colloid chemistry/nanoscience to practical application got a great impetus from National Nanotechnology Initiative issued by the US Government. Nanotechnology is defined as the understanding and control of matter at the nanoscale, at dimensions between approximately 1 and 100 nanometres, where unique phenomena enable novel applications. The manipulating of matter at this length scale opens innovation in energy, information technology, biotech, and medicine, and is finding its way into food stuffs.

Nanotechnology could offer benefits in various fields of food technology. It can bring changes in areas such as processing, storage, packaging, food safety, and development of functional foods for health promotion (MeGHANI et al., 2019). One of the first applications of nanotechnology was a carrier to deliver antimicrobial peptide required to stop the antimicrobial decay of food quality in the food industry. Starch colloid filled with the antimicrobial agent operates in a way that when microorganisms grow on the packaged food they will break the coating of starch leading to release of antimicrobial agents.

In a consequence of introduction of nanotechnology into food industry a so called nanofood has appeared. Nanofood expression describes food that has been cultivated, produced, processed, or packaged using nanotechnology techniques or tools, or to which manufactured nanomaterials have been added.

Starting from the First International Food Nanotechnology Conference (Atlanta, 2006), food industry tries to identify opportunities and the most remarkable discoveries in nanotechnology that can significantly enhance food processing and food products in the following areas:

* To whom correspondence should be addressed.

E-mail: kisseva@caesar.elte.hu 
- health promotion through bioactive compound delivery;

- ingredients with enhanced functionality, with novel flavour and texture;

- novel packaging materials;

- materials with microbial-repellent characteristics;

- advanced tools of nanosensors for food safety shelf life and quality monitoring.

It is also important to consider that those promising innovations should be cost-effective, and significant changes in current food regulations are needed in the near future (MORARU et al., 2009).

Nanotechnology in food processing can be evaluated by considering its role in the upgrading of food products in terms of texture, appearance, taste, flavour, nutritional value, food shelf-life, and smart food packaging. Beside these benefits concern might arise on safety and cost (MAGNUSON et al., 2011).

\section{Nanosize and nanostructured materials}

\subsection{Nanoencapsulation}

Development and application of nanosize and nanostructured materials represent an important field of food nanotechnology. Nanoencapsulation techniques have been implemented to improve flavour release, retention, and to deliver culinary balance. Nanocarriers have been introduced as delivery systems to carry food additives in food products without disturbing their elementary morphology (Meghani et al., 2019). The main advantage of using nanomaterials is that it can release encapsulated compounds much slower and over longer time periods, they are carriers of fragrances and flavours, and also provide a favourable improvement in the bioavailability of nutraceuticals due to their subcellular size.

Nanoencapsulating takes the process of encapsulation, a means for drug delivery pioneered by the pharmaceutical industry, and improves solubility of vitamins, antioxidants, healthy omega oil fractions, and other nutrients, while protecting the ingredient from various environmental factors, such as water, oxygen, heat, and light. It is also possible to mask undesirable taste, odour, and colour. Nanonutrient particles seem to be fully soluble and invisible in water and oil, widening the door for potential nutraceutical beverages. Nanoencapsulation is the application of encapsulation in the nanometer scale with films, layers, coating, or simply dispersion. Often the encapsulated ingredient is in the molecular or nanoscale form (GAONKAR et al., 2014).

Scaling down the size of food materials to nanosized crystals might also modify the texture of food product, creating more particles for an overall greater surface area (AGUILERA, 2014). The enhanced interfacial interaction can lead to change of functional properties of the system. As a result of incorporating the multiple water-in-oil-in-water emulsions of nanosized lipids, food spreadability and stability are improved. A low-fat mayonnaise formulation for example provides a satisfying fatty mouth feel, with the obvious benefit that extra stabilisers and thickeners are not needed to achieve the desirable texture. The nanoemulsion could have its application in formulating low-fat products.

\subsection{Main types of nanoencapsulation systems}

The main types of nanoencapsulation systems designed for food application are formed from accepted food ingredients as lipids, carbohydrates, and proteins, which are in the group of GRAS (generally regarded as safe materials). 
Liposomes are the best known lipid based delivery systems. The shell like lipid bilayer structure practically prevents the degradation of the incorporated food ingredient. Manufacture of nanoliposomes, however, requires high energy for the dispersion of lipid/phospholipid molecules in the aqueous medium. Another special lipid delivery system is the cochleate, a spiral like associate of lipid bilayer. Cochleates have a multilayered structure consisting of a large, continuous lipid bilayer sheet rolled up in a spiral, delivering their contents to target cells through the fusion between the outer layer of the cochleate and the cell membrane (SANTANGElo et al., 2000). The multi-layered structure of cochleates provides protection from degradation of the 'encochleated' molecules, even when exposed to harsh environmental conditions, allowing their transport toward the destination (Bozo et al., 2014). As liposomes, cochleates are mainly for encapsulation of water soluble bioactive materials, including protein and peptide drugs and large hydrophilic molecules, although they are also proved to be suitable carriers for compounds with poor water solubility (GOULD-FOGERITE et al., 2003). It is expected that such systems will be used in the future for the encapsulation, targeted delivery, and controlled release of chemically sensitive functional food molecules (SHIMONI, 2009).

Nanoparticles can be formed from food biopolymers, proteins, or polysaccharides by self-association, controlled aggregation, or inducing phase separation to encapsulate functional ingredients.

Polysaccharide-based biodegradable nanoparticles can be used to control rheological behaviour or for nanoencapsulation. A new beverage product was prepared applying starch, probably the most abundant functional polysaccharide in foods, by ultra-high-pressure homogenisation (CHEN \& WAGNER, 2004). The enriched product contained powder with particle size around $100 \mathrm{~nm}$ with about $15 \%$ vitamin $\mathrm{E}$ acetate, which was stable in the beverage and did not alter its appearance.

The design of protein type delivery carrier system focuses on milk or whey proteins as source materials. According to a recent concept (Sемо et al., 2006), self-assembled casein micelles are used as nanocapsular vehicles. The authors realised that casein micelles (CM) are effective nanocapsules created by nature to deliver minerals and nutrients, such as calcium, phosphate, and protein to neonates. Thus, they suggested using $\mathrm{CM}$ as a selfassembled system for nanoencapsulation and stabilisation of hydrophobic nutraceutical substances for enrichment of food products. Vitamin D2, used as a model for hydrophobic nutraceutical compounds, was successfully loaded by utilising the natural self-assembly tendency of bovine caseins. In addition to the increased vitamin concentration, a partial protection against UV light-induced degradation of vitamin D2 by the micelles was found. Casein micellar nanocapsules may be incorporated in dairy products without modifying their sensory properties (SHIMONI, 2009).

\subsection{Emulsion type systems}

Emulsion type systems are composed of phases of different polarities, therefore they can carry hydrophobic food components in aqueous phase emulsions. The main types of nanostructures formed from immiscible liquids as $\mathrm{O} / \mathrm{W}$ and $\mathrm{W} / \mathrm{O}$ emulsions, the corresponding nanoemulsions, furthermore the double emulsions, are presented in Figure 1. These systems cover a wide size range from nanometer to micrometer. As a comparison, surfactant micellar and lipid liposome systems are also included. 


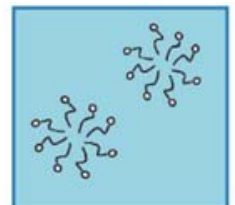

Micelle in water

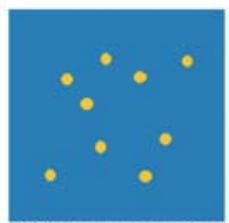

$\mathrm{O} / \mathrm{W}$ nanoemulsion

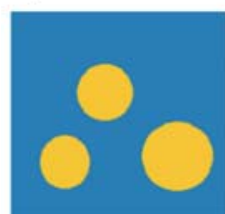

$\mathrm{O} / \mathrm{W}$ emulsion

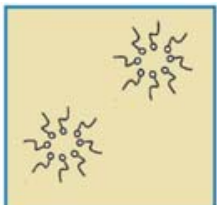

Inverse micelle in oi

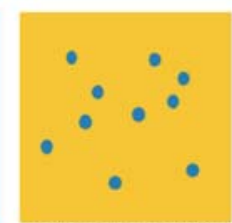

W/O nanoemulsion

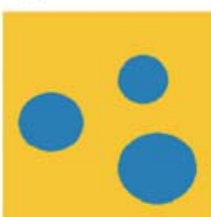

W/O emulsion
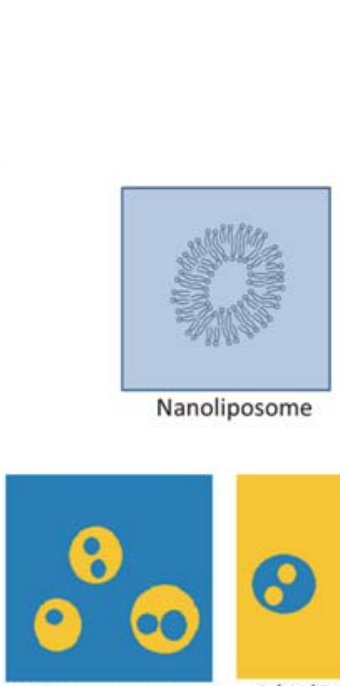

W/O/W emulsion

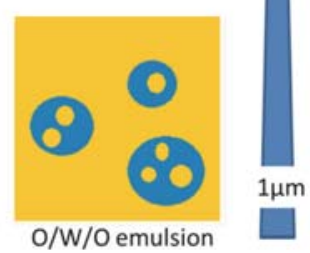

$\mathrm{O} / \mathrm{W} / \mathrm{O}$ emulsion

Fig. 1. Structure of surfactant micelles, liposome, and various types of nanoemulsions and emulsions

Milk, cream, ice-cream, dressings, mayonnaise, beverages, soups, dips, and sauces are typical examples of oil-in-water $(\mathrm{O} / \mathrm{W})$ emulsions, while butter, margarine, and some spreads are examples of water-in-oil emulsions (W/O). Common emulsions are (macro-) emulsions with a milky, opaque appearance with droplet diameters between $100 \mathrm{~nm}$ and $100 \mu \mathrm{m}$. These emulsions tend to show instability such as gravitational creaming or sedimentation, aggregation, coagulation, and Ostwald ripening finally leading to phase separation. The emulsions are also classified according to the character of the emulsifying agents such as surfactant, polymers, or particles. The application of the latter, called Pickering emulsions, relevant to food and drink was reviewed by MurRaY (2019). Organic crystals, cellulose, starch, and microgel particles from food components provide a particularly versatile and effective way of stabilisation.

On the contrary to emulsions, microemulsions are optically clear and thermodynamically stable systems with the lowest droplet size of 1-20 $\mathrm{nm}$ and ultra-low interfacial tension $\left(10^{-1}-10^{-3} \mathrm{mN} \mathrm{m}^{-1}\right)$ between the hydrophobic (oily) and the hydrophilic (aqueous) phases. Microemulsions form spontaneously by mixing water, oil, and a significant amount of surfactant. Their major disadvantage is that large quantity of surfactant is required, which can lead to problems related to flavour, cost, or legality (WeISS et al., 2006).

The system nanoemulsion is in between the microemulsion and emulsion, possessing some properties of both. The interfacial tension at the phase boundaries in nanoemulsions is defined as $1-10 \mathrm{mN} \mathrm{m}^{-1}$. Nanoemulsion can be seen transparent or semi-transparent with hazy, bluish appearance due to their small particle diameters in the range of 20-100 nm. (Nanoemulsions are also referred to in the literature as miniemulsions, ultrafine emulsions, emulsoids, unstable microemulsions, and submicrometer emulsions.) The characteristics that make nanoemulsions attractive for formulation of oil and water containing food products are the highly reduced amount of surfactant required compared to microemulsions and the 
enhanced kinetic stability compared to emulsions. Beneficial consequences of small droplet size are translucent appearance and low viscosity (Solans et al., 2005). Nanoemulsions are unique due to their long term physical stability with no apparent flocculation, coalescence, creaming, or sedimentation, hence the shelf life of such product is extended. Nanoemulsions can be used to increase the degree of dispersion of highly hydrophobic bioactive compounds. Emulsion with nanometre size droplet diameters are prepared by using high-pressure valve homogeniser, microfluidiser, or a low energy input method called phase inversion suggested recently (PorRAs et al., 2008). Therefore, nanoemulsions provide a great potential for increasing bioavailability of lipophilic functional substances. Solubilisation of phytochemicals like curcumin, resveratrol, carotenoids such as lycopene, beta-carotene, lutein, and zeaxanthin using nanoemusions was reported (HUANG et al., 2010). Bioavailability of essential oils can be increased by incorporation of the essential oils into the nanoemulsions, providing a safe alternative to synthetic additives. Due to the small droplet size and high optical clarity, there is great promise of nanoemulsions in the beverage industry, e.g. cinnamon oil based nanoemulsion to deliver vitamin D. Nanoemulsions can be a way to substitute clarifiers such as alcohol in beverage industry (YALÇINÖZ \& ERÇELEBI, 2018).

\section{Nanoencapsulation of antimicrobial agents}

The specific goal with the antimicrobials is to improve the quality and safety of foods. Among the FDA approved antimicrobials, we can find various compounds like acids (acetic, benzoic, sorbic, lactic, propionic acid), nitrite, nitrates, parabens, and even proteins such as lysozyme and nisin as well. The antimicrobials in the food system can be applied to the packaging material or/and to the food itself. Obviously, nanoencapsulation is promising in both cases. The antimicrobial nanoencapsulation system should be able to reduce the potential negative sensory effects of antimicrobials (e.g. by suppressing volatility) and to not increase (or possibly even decrease) the minimum inhibitory concentrations of antimicrobials to allow for a reduction of the required concentrations. Furthermore, the use of food antimicrobials must be economically feasible (Weiss et al. 2009).

The requirements for the nanoencapsulation systems are similar to the encapsulation of other bioactive components with special importance, controlled, gradual release of sufficient amount of antimicrobials to continuously inhibit bacterial growth. Solid lipid nanoparticles (SLN) as carriers are well suited for hydrophobic antimicrobials. The diffusion of molecules is slower than in liquid oil, so the solid matrix slows down movement of the bioactive compound to the droplet surface, as well as slows down penetration of any reactants (e.g. metals and oxygen) into the particle (LEAL-CALDERON et al., 2007). Their appearance despite the small size might be turbid, because the refractive index of solid fat is higher than liquid oil. Gravitational stability is rather good, since the density difference between the particles and the medium is small (MCCLEMENTS, 2005). Crystallised matrices can also be convenient means to building a preservation system that is able to provide long-term protection by slowly releasing the antimicrobial agent into the food. Crystalline matrices can contribute to maintaining the activity during storage by reducing the volatility of the encapsulated compounds, which is advantageous when essential oils with high volatility are applied as food antimicrobials (BURT, 2004).

Liposomes as spherical hollow particles from lipids with aqueous interior are ideal delivery systems for hydrophilic antimicrobials. Liposomes can serve as a separate 
microenvironment, in which food antimicrobials, especially protein types, can be incorporated maintaining their activity despite changes in the surrounding aqueous phase. Both nisin and lysozyme were successfully encapsulated into liposomes and were exposed to environmental and chemical stresses typically encountered in foods and food processing operations. It was found that liposomes containing nisin may be suitable for use as antimicrobial-active ingredients in low- or high-pH foods subjected to moderate heat treatments (TAYLOR et al., 2007). However, the main concern with liposomes is the lack of system stability. That can be overcome by formation of a coating of biopolymers on the surface of liposomes. A variety of polymers such as chitosan, $\beta$-lactoglobulin, fish gelatine, and casein were adsorbed on the surface of liposomes resulting in dramatic improvement in the long-term stability of the particles (LAYE et al., 2008). Additionally, surface modification with cationic polymers provides a possibility to improve electrostatic interaction of the liposome with the target bacterial cell surface (CARUSO \& MOHWALD, 1999), which may have an influence on antimicrobial activity.

Microemulsions are thermodynamically stable systems containing large amount of surfactant, with a high chance to keep its highly dispersed structure upon dilution. A series of investigations on the use of microemulsions to deliver food antimicrobials and inhibit the growth of foodborne pathogens were conducted. GAYSINSKY and co-workers (2005) reported $0.9 \%$ eugenol encapsulation into micelles at a surfactant (Surfynol $485 \mathrm{~W}$ ) concentration of a few percent. Growth inhibition of four strains of Escherichia coli and Listeria monocytogenes by eugenol in microemulsions incubated at various temperatures was determined. Eugenol encapsulated in surfactant micelles proved to be effective in inhibition of both microorganisms at $\mathrm{pH} 5,6$, and 7 .

Nanoemulsions are also attractive delivery systems for antimicrobials due to their versatile character to accept both hydrophilic and hydrophobic type of antimicrobials, and also because of their significant stability. When droplet size falls below a critical value (d $100 \mathrm{~nm}$ ), emulsions become stable to creaming or sedimentation, because the effects of Brownian motion dominate gravitational effects (MCCLEMENTS, 2005). Nanoemulsions can thus be kinetically stable for many years. Nanoemulsion formation needs less amount of surfactant than microemulsions, but the choice of surfactant is critical, since emulsifiers have to rapidly cover the many new surfaces that are formed. Generally, two classes of surfaceactive species are used: (1) small-molecule surfactants such as monoglycerides, sucrose esters, and others and (2) macro-molecular emulsifiers such as proteins or modified starches (Dickinson, 2003). Since small-molecule surfactants adsorb much faster to newly formed interfaces than macromolecular surfactants, they are typically better suited for manufacturing nanoemulsions. The appearance of emulsions is strongly dependent on droplet size, and emulsions become transparent, when the size of the droplet falls below a critical diameter $(\mathrm{d}<90-100 \mathrm{~nm})$, allowing their application in clear beverage products.

\section{Nanoencapsulation of vitamins}

Nanoencapsulation of vitamins with different techniques is expected to be a crucial field of research and development of enriched human food (KATOUZIAN \& JAFARI, 2016). Vitamins are bioactive molecules necessary for human health, which are sensitive to degradation. During consumption, the bioavailability of these compounds might be limited due to their structural change or low absorption. By entrapping vitamins in the nanocapsules, excellent 
bioavailability for both water soluble and oil soluble vitamins can be achieved. Nanoencapsulation is a promising approach for targeted delivery and controlled release of vitamins. Such products possess various benefits, including the protection of vitamin molecules from destructive environment during processing and delivery, masking the possible undesirable flavour, and long shelf life due to their increased colloid stability. The nanoparticle type carrier allows the implementation of enhanced beneficial effect of vitamins, since the release of the active component is controlled, and targeting and high cellular uptake are enabled.

Nanoencapsulation-based technologies are emerging research areas in the food industry, employing different nanocarriers like nanoparticles, nanoliposomes, and nanoemulsions. These techniques allow development of efficient nanoencapsulation of both hydrophilic and lipophilic vitamins, applying commercially approved compounds, including natural biopolymers such as starches, cyclodextrins, alginates, proteins, and also GRAS lipids and surfactants. The nanosystems encapsulating oil- and water soluble vitamins are collected in Table 1.

Table 1. Nanoencapsulation of oil- and water soluble vitamins

\begin{tabular}{|c|c|c|}
\hline Vitamin & Encapsulation system & Reference \\
\hline \multicolumn{3}{|l|}{ Oil soluble vitamins } \\
\hline $\mathrm{D}$ & Nanoemulsion & GuTtoFf et al., 2015 \\
\hline D3 & Protein nanoparticle & $\begin{array}{l}\text { ABBASI et al., 2014; LeE et al., 2016; } \\
\text { DAVID \& LiVNEY, } 2016\end{array}$ \\
\hline D2 & Casein micelle & SEMO et al., 2006 \\
\hline D2 & Solid lipid nanoparticle & PATEL et al., 2012 \\
\hline E acetate & Nanoemulsion & $\begin{array}{l}\text { SABERI et al., 2013; OzTURK et al., } \\
\text { 2014; DASGUPTA et al., 2016; } \\
\text { HATEGEKIMANA et al., } 2015\end{array}$ \\
\hline E acetate & Polysaccharide nanoparticles & CHEN \& WAGNER, 2004 \\
\hline $\mathrm{E}$ & Liposome & $\begin{array}{l}\text { MARSANASCO et al., 2011, LIU \& PARK, } \\
2009\end{array}$ \\
\hline Lycopene, carotene, lutein & Chitosome (chitosane coated liposome) & TAN et al., 2016 \\
\hline \multicolumn{3}{|l|}{ Water soluble vitamins } \\
\hline B9 & Nanoemulsion, double emulsion & ASSADPOUR et al., 2016 \\
\hline $\mathrm{B} 2$ & Double emulsion & Bou et al., 2014 \\
\hline $\mathrm{C}$ & Liposome & MARSANASCO et al., 2011 \\
\hline B12 & Small unilamellar vesicle & BochicCHIO et al., 2016 \\
\hline
\end{tabular}

Oil-in-water $(\mathrm{O} / \mathrm{W})$ nanoemulsions as delivery systems for drugs, vitamins, and nutraceuticals within functional food products are gaining increasing attention. The $\mathrm{O} / \mathrm{W}$ nanoemulsions are particularly suitable for encapsulation of lipophilic nutraceuticals because of their ability to form stable and transparent delivery systems with high oral bioavailability. Vitamin $\mathrm{D}_{3}$ was entrapped in whey protein isolate (WPI) nanoparticles and the stability was investigated in presence of air for 7 days (ABBASI, 2014). Particles formed compact structures in the presence of calcium, and the oxygen diffusion into the particle was inhibited. The results illustrate that these nanoparticles can be used either in clear or non-clear beverages as 
enriching agent. LEE and co-workers (2016) applied another protein isolate obtained from soy to prepare nanoemulsion to deliver vitamin $\mathrm{D}_{3}$. The oil droplet size was in the range of $70-120 \mathrm{~nm}$, while the protein nanoaggregates provided good protection of vitamin $\mathrm{D}_{3}$ against UV exposure $(180 \mathrm{~min}$ ), with retention above $70 \%$, compared to $5.2 \%$ in the control. The advantage of spontaneous emulsification method to prepare nanoemulsion for vitamin $\mathrm{D}$ was presented by GutTOFF and co-workers (2015). The influence of oil phase composition, surfactant type (Tween 20, 40, 60, 80, and 85), and stirring conditions on the initial particle size of vitamin D nanoemulsions was studied. Nanoemulsions with small droplet diameters $(\mathrm{d}<200 \mathrm{~nm})$ could be formed using Tween 80 at high stirring speed $(800$ r.p.m.). These systems were relatively stable to droplet growth at ambient temperature $(<10 \%$ in diameter after 1 month storage). The thermal stability of the nanoemulsions could be improved by adding a cosurfactant, sodium dodecyl sulphate. The spontaneous emulsification method has great potential in forming nanoemulsion-based delivery systems for food and other applications, because it is simple and inexpensive. Spontaneous emulsification was also used for encapsulation of vitamin E acetate (SABERI et al., 2013). The effect of cosolvents, propylene glycol (PG) and ethanol, on the formation, stability, and physical properties of vitamin E loaded nanoemulsion was studied. The particle size and optical clarity of the nanoemulsions (10\% vitamin $\mathrm{E}+10 \%$ surfactant $+80 \%$ aqueous phase) depended strongly on cosolvent type and concentration. The smallest droplets $(\mathrm{d}<50 \mathrm{~nm})$ and highest transparency were observed when either $30 \%$ PG or $20 \%$ ethanol was present in the aqueous phase. Dilution of the nanoemulsions $(100 \times$ with water $)$ prior to storage considerably improved their long-term stability, especially at higher storage temperatures. Vitamin E acetate loaded nanoemulsion was successfully formed using natural small molecule surfactants: quillaja saponin and lecithin (OzTURK et al., 2014). The effect of oil composition (0-100\% vitamin E acetate), surfactant type, and surfactant concentration $(0.0005-5 \%)$ on the mean particle diameter and vitamin loading capacity was examined. The mean particle diameter had a minimum value (about $120 \mathrm{~nm}$ ) at an intermediate vitamin E-to-orange oil ratio. The mean particle diameter decreased with increasing surfactant concentration for both surfactants. Both systems were found to be stable at temperatures from 30 to $90{ }^{\circ} \mathrm{C}$ (pH 7). A food grade vitamin $\mathrm{E}$ acetate nanoemulsion using edible mustard oil and surfactant Tween 80 was prepared by DASGUPTA and co-workers (2016). A stable nanoemulsion for 15 days with droplets of spherical morphology and a size distribution of $86.45 \pm 3.61 \mathrm{~nm}$ was obtained. These nanoemulsions showed improved bio-, antioxidant, and antimicrobial activities and could be potentially used to increase the shelf life of fruit juice. Beside the stability of the nanoemulsion, vitamin $\mathrm{E}$ degradation was also characterised under a wide range of environmental conditions by HATEGEKIMANA and co-workers (2015). The low energy emulsification method known as Emulsion Phase Inversion was applied with different carrier oils (short-chain, medium-chain, and long-chain triglycerides) and Tween 80 as colloid stabiliser. Although nanoemulsions made with vitamin $\mathrm{E}$ and the three carrier oils showed physical stability to heat shock $\left(30-90^{\circ} \mathrm{C}, 30 \mathrm{~min}\right)$, ionic strength $(0-500 \mathrm{mM}), \mathrm{pH}(2.0-$ 8.5 ), and long term storage (60 days, under light and darkness, 4,25 and $40{ }^{\circ} \mathrm{C}$ ), there was significant vitamin $\mathrm{E}$ degradation in heat processed and long-term stored samples. Vitamin $\mathrm{E}$ retention increased when the nanoemulsions were stored in the dark.

The main purpose of nanoencapsulation is the protection of the bioactive component in the case of water soluble vitamins. The inverse type emulsion, water in oil (W/O), is suitable to encapsulate the various hydrophilic B vitamins. Double emulsion $(\mathrm{W} / \mathrm{O} / \mathrm{W})$ is the perfect form to easily introduce an aqueous system into e.g. beverages. Water in oil nanoemulsions 
containing folic acid (vitamin $\mathrm{B}_{9}$ ) was prepared and optimised by a low energy (spontaneous) emulsification technique using a non-ionic surfactant (Span 80) (AssadPour et al., 2016). The optimum nanoemulsion formulation with $12 \%$ dispersed phase, a water to surfactant ratio of 0.9 , and folic acid content of $3 \mathrm{mg} \mathrm{ml}^{-1}$ in dispersed phase was encapsulated within maltodextrin-whey protein double emulsions. Droplet size decreased at higher surfactant contents, and the final nanoemulsions had a droplet size $<100 \mathrm{~nm}$. Bou and co-workers (2014) examined the influence of the type of lipid source (chia oil, sunflower oil, olive oil, or rendered pork backfat) on the physicochemical properties of riboflavin (vitamin $\mathrm{B}_{2}$ ) encapsulation in food-grade $\mathrm{W} / \mathrm{O} / \mathrm{W}$ double emulsions for use as functional healthier-fat food ingredients. Thermal treatment caused minimal changes in oil droplet particle size characteristics, viscosity, dynamic rheological properties, physical stability, and encapsulation efficiency. Riboflavin was efficiently encapsulated, and the carrier system was stable to environmental stresses typically occurring in the food industry.

In recent years, much attention has been given to lipid carriers as potential delivery systems for bioactive molecules, due to their favourable properties such as high biocompatibility, size and composition versatility.

Nanoliposomes provide excellent possibility to encapsulate both hydrophilic and hydrophobic compounds. The inner core of the nanovehicle is an aqueous phase, which can deliver a relative high amount of water soluble vitamin, while the nonpolar section of the lipid bilayer can incorporate hydrophobic, oil soluble vitamins. For this purpose, multilayer liposomes offer higher encapsulation capacity. Soy phosphatidylcholine-based liposomes were developed as vitamin transporters for vitamins $\mathrm{E}$ and $\mathrm{C}$ to be incorporated in orange juice (MARSANASCO et al., 2011). Liposomes had a protective effect on antioxidant activity of vitamins before and after pasteurisation. Addition of stearic acid favours oxidative stability and encapsulation efficiency due to a bilayer stabilising effect. It is important to note that the combination of liposomal formulations and vitamins with orange juice did not change its organoleptic characteristics and showed microbiological stability after pasteurisation and storage at $4{ }^{\circ} \mathrm{C}$ for 37 days. An innovative ultrasound-assisted approach was described by BochicCHIO and co-workers (2016) to obtain nanostructures (Small Unilamellar Vesicles, SUVs) less than $100 \mathrm{~nm}$ in size, loaded with different vitamins (B12, tocopherol and ergocalciferol). SUVs with diameter size ranging from $40 \mathrm{~nm}$ to $51 \mathrm{~nm}$ were obtained starting from MLVs with a diameter range of 2.9-5.7 $\mu \mathrm{m}$. Stability tests have shown that the used lipid composition allows keeping the nanovesicles and their content intact for more than 10 days when incubated under simulated extracellular environmental conditions.

A new nutraceutical carrier, chitosan-coated nano-size liposome from phosphatidylcholine (pc) and cholesterol was developed by LIU and PARK (2009). Sonication method was applied to get small liposomes with mean diameter $\sim 82 \mathrm{~nm}$. Chitosan-coated nano-size liposomes appeared to be promising vitamin E carriers, with the highest payload over $27 \%$. The stability of the suspension of loaded liposomes during the 8 weeks of storage was over $90 \%$ under $4^{\circ} \mathrm{C}$. The previously described cithosan coated nano liposome, the chitosome, is the delivery system for various carotenoids (lycopene, $\beta$-carotene, lutein, and canthaxanthin) in the report of TAN and co-workers (2016). It was revealed by fluorescence analysis that the interaction of chitosan with liposomal membrane, such as electrostatic and hydrophobic interaction, restricted the motion freedom of lipid molecules and enhanced their order at the polar headgroup region and hydrophobic core of the membrane. These rigidifying effects were directly responsible for the stabilisation of carotenoid-loaded liposomes against heat and gastrointestinal stress. The liposomal membrane was able to protect $\beta$-carotene and lutein 
to a higher extent than lycopene and canthaxanthin. These results may contribute to the development of chitosomes as potential candidates for an efficient delivery of bioactive compounds in nutraceutical and functional foods.

The use of solid lipid nanoparticles (SLNs) is a technique that has been widely used in the pharmaceutical industry for the last 2 decades and has become of increasing interest to food scientists due to its potential for encapsulation and controlled release. The classical lipid based carrier, the lipid nanoparticle, is usually the solidified form of $\mathrm{O} / \mathrm{W}$ emulsion droplet. Starting from nanoemulsion nano sized solid crystalline lipid spheres can be obtained containing the bioactive compound dispersed in the matrix. Encapsulation of ergocalciferol (vitamin $\mathrm{D}_{2}$ ) performed in tripalmitin SLNs stabilised by polysorbate 20 (Tween 20) was reported by PATEL and co-workers (2012). They found that, with increased vitamin $\mathrm{D}_{2}$ concentration, the clarity of the dispersion increased, and the stability of the lipid crystal structure was affected in a way that indicated a higher capacity of the lipid to incorporate vitamin molecules and hence, to protect them better from oxygen and light. That vitamin loaded SLNs offer a useful way for fortification of clear juices with ergocalciferol. Another solution was proposed by DAVID and LIVNEY (2016) for fortifying beverages with vitamin D of low water solubility and acid-sensitivity. Potato protein, a widely available and inexpensive raw material, considered GRAS and non-allergenic was used. Vitamin D3 - potato protein

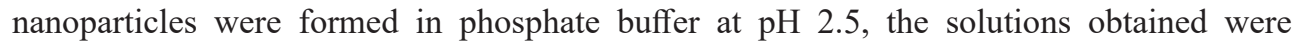
transparent. Nanocomplexation provided significant protection and reduced vitamin losses during pasteurisation and simulated shelf life tests under several different sets of storage conditions. Hence, potato protein shows promise as a good protective carrier for Vitamin D3, and possibly other hydrophobic bioactives, for enrichment of clear beverages and other food $\&$ drink products, to promote human health.

\section{Texture modification}

To provide elderly people with healthy and soft food is recently an essential task for the food industry. The need for texture modified food products becomes more demanding with the fast growing rate of older people in the population (AguiLERA \& PARK, 2016). Nanotechnology might offer a valuable contribution to the development and introduction of tailor-made soft foods. Texture-modified foods (TM) is a term that refers to foods with soft-textures and/or reduced particle size as well as thickened liquids (drinks) aimed at the market segment of seniors with eating dysfunctions. Categories of soft and TM food are thin drinks, soups, thickened liquids, creamy food jellies, mousses, purees, and pates, minced and moist foods, and process-softened foods (ZARGARAAN et al., 2013). Older people are particularly exposed to nutritional deficiencies, so specific recommendations have been proposed for this age group. Higher daily protein, vitamin D, and calcium intake, soft, fibre-rich food, which is tasty and easy to safely swallow are required.

Considering the technologies, some conventional methods can be used to soften food and meals, freeze-thawing, enzyme impregnation, high-pressure processing, use of pulsed electric fields, and sonication. Proper control of process variables allows preserving the colour and flavour of food products, while tuning their soft texture to different extents. Additionally, there are novel technologies, microfluidics (MARQUIS et al., 2014), 3D printing (Godor et al., 2016), electrospinning (GHORANI \& TUCKER, 2015), elecrospraying (GomeZMASCARAQUe et al., 2015) leading to small particles, which may find applications in TM 
foods. Proteins, carbohydrates, and lipids are the basic building blocks for the design of TM foods. Globular proteins unfold and denature when heated, increasing the viscosity of liquids (e.g. in protein drinks). On further heating they may self-assemble into nano-sized aggregates and fibrils, eventually becoming the network chains of gels. Polysaccharides are used to thicken aqueous food dispersions, stabilise emulsions and foams, and as gelling agents. Gums and starches are often used as thickening agents and encapsulating matrices for nutrients, antioxidants, colorants, enzymes, and flavours. Monoglycerides and phospholipids may be used as emulsifiers in interfaces due to their amphiphilic nature or self-associated into a multitude of nanosized structures (e.g., micelles and vesicles) as delivery vehicles for nutrients and bioactives.

Due to their small and tuneable size, soft texture, and free flowing state, gel microparticles are excellent alternatives to tailor the rheological properties of foods. They may be mixed into thin liquids or added to purees to modify their flow behaviour and texture perception. Loaded with flavours and provided with weak and fragile texture, they elicit stronger aroma feel during breakdown in the mouth.

Feeding the elderly with tasty and nutritious food is considered a major challenge for the food industry in many countries of the world.

\section{Nanotechnology in food packaging}

Consumers' expectation towards food packaging is to provide food safety and to maintain food freshness and sensory values. A further convenience for the seller and the customer is to get information on the history of thermal or other environmental conditions possibly influencing the quality of food products during their shelf life. That demand could be met by various nanostructural sensors and indicators built into smart packaging materials (OzDEMIR \& KeMERLI, 2016).

According to the legal definition of the EU (EC, 2009), intelligent packaging contains a component that enables the monitoring of condition of the packaged food or the environment surrounding the food during transport and storage. That provides the user with reliable and correct information on the conditions of the food, environment, or package integrity (VANDERROOST et al., 2014). Active packaging is an extension of the protection function of traditional food packaging, and is designed such that it contains a component that enables the release or adsorption of substances into food or the environment. In such a system the product, the package, and the environment interact in a positive way to extend shelf life, improve the condition of the packaged food. Both packing systems can work synergistically to realise the so-called smart packaging. Smart packaging provides a complete packaging solution that on the one hand monitors changes in the product or the environment and on the other hand acts upon these changes. This kind of closed-loop packaging system offers the perspective to monitor changes in the product, packaging, and/or the environment and to respond appropriately on these changes via a feedback mechanism.

Such innovative approach of development of packaging system with novel functionality can be the answer to the emerging need to solve safety and quality issues through the supply chain and reduce product losses. The application of micro- and nanoencapsulation covers the area of designing food packaging loaded with bioactive components, the time and temperature indicators, as well as pressure triggered fragrance and flavour releasing systems. The active components of these intelligent packaging materials provide important functions as 
antimicrobial, antioxidative, or even insect or rodent repellent properties to enhance the safety of food products. This requires appropriate technologies that can be integrated into food packaging preserving the quality of packaged food and comprising the concept of controlled release. The most important positive impact on food quality and storage stability is that the amount of preservatives applied could be greatly reduced (OzDEMIR \& KEMERLI, 2016). Encapsulation of antimicrobial agents represents some of the earlier applications of active packaging technology (LACosTE et al., 2005; CHEN et al., 2012). Foods such as cheese, sliced meat, and bakery that are prone to spoiling on the surface have been the focus for contact packaging imbued with antimicrobials. A food film for bakery products that incorporates the essential oil of cinnamon was brought to market. Working with oregano oil and apple puree, scientists created edible food films that are able to kill certain E. coli. Packaging made with nano particles of zinc, calcium, magnesium oxide, and titanium dioxide, materials that are cheaper than nano silver, were tested as antimicrobials and were found effective.

Another reason behind the possible deterioration of food is oxygen. Oxygen inside the food packaging causes oxidation of fats and oils. It is also responsible for discoloration, changes in texture, rancidity, off-odour, and flavour problems (MEGHANI et al., 2019). Utilisation of nanomaterial helps to enhance the safety of food by avoiding spoilage or loss of food nutrients. Nanoclays embedded in plastic bottles and nylon food films stiffen packaging and reduce gas permeability. The nano-enhanced barrier keeps oxygen-sensitive foods fresher and can reduce packaging costs for manufacturers. A low cost nanoclay composite interior coating for paperboard cartons was created to keep juice fresher. Nanocomposites with oxygen radical scavenging ability given to plastic bottles offer a 6-month shelf life for beer. In another development of composite packaging material, addition of 3-5\% nanoclay to plastic significantly improved the oxygen, carbon dioxide, moisture, and volatile barrier properties as well as heat-resistance and strength of plastic beer bottles. Starch/clay and hydroxyapatite/chitosan are further nanocomposite materials of natural components with particularly good barrier properties, application of which could enhance the shelf life of foods such as processed meat, cereals, and cheese (MorARU et al. 2009).

Beside the quality preserving packaging, the monitoring of products is a major focus of food-industry-related nanotechnology. Such systems are able to respond to environmental conditions and alert the customer if the food is contaminated. With embedded nanosensors in the packaging, consumers will be able to read the condition of the food inside. The indicators provide immediate visual, qualitative information about the packaged food by means of a colour change, an increase in colour intensity, or diffusion of a dye along a straight path. Changes should be irreversible. Researchers are continuously searching for sensitive tools that are fast, accurate, and ultrasensitive to detect foodborne pathogens and their toxins. The possibility to combine biology and nanoscale technology into sensors holds the potential of increased sensitivity and reduced response time. Bioanalytical nanosensors would be able to detect a single virus particle long before the virus multiplied and long before symptoms are evident (FARHANG, 2009).

Sensors offer the prospect to provide an alternative to the time consuming, expensive, and destructive analytical techniques that are currently applied to monitor a packaged food product and its environment throughout the entire supply chain, though there is still a need to overcome the present limitations of sensors. The flexible printed sensors might be a promising alternative to silicon based systems. They are lightweight, bendable, rollable, large area, thin, small, sensitive, selective, and reversible with similar or better optical and electrical 
characteristics. By connecting one or more sensors to a radiofrequency identification tag, ensuring energy supply and storage of data in the memory, important historical information can be gathered on the quality status of the food. This remote and non-destructive way of monitoring food products is especially relevant for the transport and storage of perishable goods such as fruit, vegetables, meat, and fish.

The environmental aspect of food packaging is also crucial, as innovations should serve the reduction of harmful impact of packaging waste and food loss on our environment (VANDERroost et al., 2014).

This work was completed in the ELTE Institutional Excellence Program (NKFIH-1157-8/2019/FEKUTSTRAT) supported by the Hungarian Ministry of Human Capacities.

\section{References}

Abbasi, A., Emam-Djomeh, Z., Mousavi, M.A.E. \& Davoodi, D. (2014): Stability of vitamin D 3 encapsulated in nanoparticles of whey protein isolate. Food Chem., 143, 379-383.

Aguilera, J.M. (2014): Where is the nano in our foods? J. Agr. Food Chem., 62, 9953-9956.

Aguilera, J.M. \& PARK, D.J. (2016): Texture-modified foods for elderly: Status, technology and opportunities. Trends Food Sci. Tech., 57, 156-164.

Assadpour, E., Maghsoudlou, Y., Jafari, S.-M., Ghorbani, M. \& Aalami, M. (2016): Optimization of folic acid nano-emulsification and encapsulation by maltodextrin-whey protein double emulsions. Int. J. Biol. Macromol., 86, 197-207.

Bochicchio, S., Barba, A.A., Grassi, G. \& Lamberti, G. (2016): Vitamin delivery: Carriers based on nanoliposomes produced via ultrasonic irradiation. LWT-Food Sci. Technol., 69, 9-16.

Bou, R., Cofrades, S. \& Jimenez-Colmenero, F. (2014): Physicochemical properties and riboflavin encapsulation in double emulsions with different lipid sources. LWT-Food Sci. Technol., 59, 621-628.

Bozo, T., Brecska, R., Gróf, P. \& Kellermayer, M.S.Z. (2014): Extreme resilience in cochleate nanoparticles. Langmuir, 31(2), 839-845.

BuRT, S. (2004): Essential oils: Their antimicrobial properties and potential applications in_food - A review. Int. J. Food Microbiol., 94, 223-253.

Caruso, F. \& Mohwald, H. (1999): Preparation and characterization of ordered nanoparticle and polymer composite multilayers on colloids. Langmuir, 15(23), 8276-8281.

Chen, C.-C. \& Wagner, G. (2004): Vitamin E nanoparticle for beverage applications. Chem. Eng. Res. Des., 82(A11), 1432-1437.

Chen, X., Lee, D.S., Zhu, X. \& Yam, K.L. (2012): Release kinetics of tocopherol and quercetin from binary antioxidant controlled-release packaging films. J. Agr. Food Chem., 60, 3492-3497.

Dasgupta, N., Ranjan, S., Mundra, S., Ramalingam, C. \& Kumar, A. (2016): Fabrication of food grade vitamin E nanoemulsion by low energy approach, characterization and its application. Int. J. Food Prop., 19, 700-708.

DAVID, S. \& LiVneY, Y.D. (2016): Potato protein based nanovehicles for health promoting hydrophobic bioactives in clear beverages. Food Hydrocolloid., 57, 229-235.

Dickinson, E. (2003): Hydrocolloids at interfaces and the influence on the properties of dispersed systems. Food Hydrocolloid., 17, 25-39.

Farhang, B. (2009): Nanotechnology and applications in food safety. -in: Barbosa-Canovas, G., Mortimer, A., Lineback, D., Spiess, W., Buckle, K. \& Colonna, P. (Eds) Global issues in food science and technology. Elsevier, Oxford, UK, pp. 401-410.

Gaonkar, A.G., Vasisht, N., Khare, A.R. \& Sobel, R. (Eds) (2014): Microencapsulation in the food industry. Academic Press, Amsterdam. 590 pages.

Gaysinsky, S., Davidson, P.M. \& Bruce, B.D. \& Weiss, J. (2005): Stability and antimicrobial efficiency of eugenol encapsulated in surfactant micelles as affected by temperature and pH. J. Food Protect., 68(7), 1359-1366.

Ghorani, B. \& Tucker, N. (2015): Fundamentals of electrospinning as a novel delivery vehicle for bioactive compounds in food nanotechnology. Food Hydrocolloid., 51, 227-240.

Godoi, F.C., Prakash, S. \& Bhandari, B.S. (2016): 3D printing technologies applied for food design: Status and prospects. J. Food Eng., 179, 44-54.

Acta Alimentaria 49, 2020 
Gomez-Mascaraque, L.G., Lagaron, J.M. \& Lopez-Rubio, A. (2015): Electrosprayed gelatine submicroparticles as edible carriers for the encapsulation of polyphenols of interest in functional foods. Food Hydrocolloid., 49, $42-52$.

Gould-Fogerite, S., Mannino, R.J. \& Margolis, D. (2003): Cochleate delivery vehicles: Applications to gene therapy. Drug. Deliv. Technol., 3(2), 40-47.

Guttoff, M., SAberi, A.H. \& McClements, D.J. (2015): Formation of vitamin D nanoemulsion-based delivery systems by spontaneous emulsification: factors affecting particle size and stability. Food Chem., 171, 117122.

Hategekimana, J., Chamba, M.V., Shoemaker, C.F., Majeed, H. \& Zhong, F. (2015): Vitamin E nanoemulsions by emulsion phase inversion: Effect of environmental stress and long-term storage on stability and degradation in different carrier oil types. Colloids Surf. A: Physicochem. Eng. Asp., 483, 70-80.

HuAnG, Q., Yu, H. \& Ru, Q. (2010): Bioavailability and delivery of nutraceuticals using nanotechnology. J. Food Sci., 75, R50-R57.

KatOUZIAN, I. \& JAFARI, S.M. (2016): Nano-encapsulation as a promising approach for targeted delivery and controlled release of vitamins. Trends Food Sci. Tech. 53, 34-48

LaCoste, A., Schaich, K.M., Zumbrunnen, D. \& Yam, K.L. (2005): Advancing controlled release packaging through smart blending. Packag. Technol. Sci., 18, 77-87.

Laye, C., McClements, D.J. \& Weiss, J. (2008): Formation of biopolymer-coated liposomes by electrostatic deposition of chitosan. J. Food Sci., 73(5), N7-N15.

Leal-Calderon, F., Thivilliers, F. \& Schmitt, V. (2007): Structured emulsions. Curr. Opin. Colloid In., 12(4-5), 206-212.

Lee, H., Yildiz, G., dos Santos, L., Jiang, S., Andrade, J., ... \& Feng, H. (2016): Soy protein nano aggregates with improved functional properties prepared by sequential $\mathrm{pH}$ treatment and ultrasonication. Food Hydrocolloid., 55, 200-209.

LiU, N. \& PARK, H.-J. (2009): Chitosan-coated nanoliposome as vitamin E carrier. J. Microencapsul., 26, $235-242$.

Magnuson, B.A., Jonaitis, T.S. \& CARD, J.W. (2011): A brief review of the occurrence, use, and safety of foodrelated nanomaterials. J. Food Sci., 76, R126-R133.

Marquis, M., Davy, J., Cathala, B., Fang, A. \& Renard, D. (2014): Microfluidics assisted generation of innovative polysaccharide hydrogel microparticles. Carbohyd. Polym., 116, 189-199.

Marsanasco, M., Marquez, A.L., Wagner, J.R., Alonso, S.D.V. \& Chiaramoni, N.S. (2011): Liposomes as vehicles for vitamins $\mathrm{E}$ and $\mathrm{C}$ : An alternative to fortify orange juice and offer vitamin $\mathrm{C}$ protection after heat treatment. Food Res. Int., 44, 3039-3046.

McClements, D.J. (2005): Food emulsions: Principles, practice, and techniques. CRC Press, Boca Raton, 690 pages.

Meghani, N., Dave, S. \& Kumar, A. (2019): Nanofood and its application - A review. BAOJ Nanotech., 5(1), 021, $1-16$.

Moraru, C., Huang, Q., Takhistov, P., Dogan, H. \& Kokini, J. (2009): Food nanotechnology: Current developments and future prospects. Barbosa-Canovas, G., Mortimer, A., Lineback, D., Spiess, W., Buckle, K. \& Colonna, P. (Eds) Global issues in food science and technology. Elsevier, Oxford, UK, pp. 369-400.

Murray, B.S. (2019): Pickering emulsions for food and drinks. Curr. Opin. Food Sci., 27, 57-63.

OzDEMIR, M. \& KEMERLI, T. (2016): Innovative applications of micro and nanoencapsulation in food packaging. -in: LAKKIs, J.M. (Ed.) Encapsulation and controlled release technologies in food systems. Wiley Blackwell, Chichester, UK. pp. 333-368.

Ozturk, B., Argin, S., Ozilgen, M. \& McClements, D.J. (2014): Formation and stabilization of nanoemulsion based vitamin E delivery systems using natural surfactants. J. Food Eng., 142, 57-63.

Patel, M.R., Martin-Gonzalez, S. \& Fernanda, M. (2012): Characterization of ergocalciferol loaded solid lipid nanoparticles. J. Food Sci., 77, N8-N13.

Porras, M., Solans, C., González, C. \& Gutiérrez, J.M. (2008): Properties of water-in-oil (W/O) nano-emulsions prepared by a low-energy emulsification method. Colloids Surf. A. Physicochem. Eng. Asp., 324, 181-188.

SABeri, A.H., FAnG, Y. \& MCClements, D.J. (2013): Fabrication of vitamin E-enriched nanoemulsions by spontaneous emulsification: Effect of propylene glycol and ethanol on formation, stability, and properties. Food Res. Int., 54, 812-820.

Santangelo, R., Paderu, P., Delmas, G., Chen, Z. W., Mannino, R., ... \& Perlin, D.S. (2000): Efficacy of oral cochleate-amphotericin B in a mouse model of systemic candidiasis. Antimicrob. Agents Ch., 44(9), 23562360 . 
Semo, E., Kesselman, E., Danino, D., \& Livney, Y.D. (2006): Casein micelle as a natural nano-capsular vehicle for nutraceuticals. Food Hydrocolloid., 21(5-6), 936-942.

Shimoni, E. (2009): Nanotechnology for foods: Delivery systems. -in: Barbosa-Canovas, G., Mortimer, A., Lineback, D., Spiess, W., Buckle, K. \& Colonna, P. (Eds) Global issues in food science and technology. Elsevier, Oxford, UK, pp. 411-424.

Solans, C., Izquierdo, P., Nolla, J., Azemar. N.\& Garcia-Celma, M J. (2005): Nano-emulsions. Curr. Opin. Colloid In., 10, 102-110.

Tan, C., Feng, B., Zhang, X., XIA, W. \& XiA, S. (2016): Biopolymer-coated liposomes by electrostatic adsorption of chitosan (chitosomes) as novel delivery systems for carotenoids. Food Hydrocolloid., 52, 774-784.

TAYlor, T.M., Gaysinksy, S., Davidson, P.M., Bruce, B.D. \& Weiss, J. (2007): Characterization of antimicrobial bearing liposomes by zeta-potential, vesicle size and encapsulation efficiency. Food Biophys., 4, 1-9.

Vanderroost, M., Ragaert, P., Devlieghere, F. \& De Meulenaer, B. (2014): Intelligent food packaging: The next generation. Trends Food Sci. Tech., 39, 47-62.

Weiss, J., Takhistov, P. \& McClements, D.J. (2006): Functional materials in food nanotechnology. Scientific Summary. J. Food Sci., 71(9), R107-R116.

Weiss, J., Gaysinsky, S., Davidson, M. \& McClements, J. (2009): Nanostructured encapsulation systems: Food antimicrobials. -in: Barbosa-Canovas, G., Mortimer, A., Lineback, D., Spiess, W., Buckle, K. \& Colonna, P. (Eds) Global issues in food science and technology. Elsevier, Oxford, UK, pp. 425-479.

YAlÇINÖZ, S. \& ERÇELEBI, E. (2018): Potential applications of nano-emulsions in the food systems: an update. Mater. Res. Express, 5(6), 062001

Zargaraan, A., Rastmanesh, R., Fadavi, G., Zayeri, F. \& Mohammadifar, M.A. (2013): Rheological aspects of dysphagia-oriented food products: A mini review. Food Sci. Hum.Wellness, 2(3-4), 173-178.

Open Access statement. This is an open-access article distributed under the terms of the Creative Commons Attribution 4.0 International License (https://creativecommons.org/licenses/by/4.0/), which permits unrestricted use, distribution, and reproduction in any medium, provided the original author and source are credited, a link to the CC License is provided, and changes - if any - are indicated. (SID_1) 